\title{
POINCARÉ DUALITY AND COBORDISM
}

\author{
BY \\ R. LASHOF
}

A smooth manifold $M^{n}$ imbedded in $(n+2 r)$-dimensional space $E^{n+2 r}$ is called weakly complex if a specific reduction of the normal bundle to the unitary group $U_{r}$ is given. A 'complex cobordism theory' for such manifolds may be defined and Milnor [4] has shown that two weakly complex closed manifolds belong to the same cobordism class if and only if they have the same Chern numbers.

The following theorem says roughly we may kill a nonzero multiple of a characteristic class of a closed weakly complex manifold that does not show up in the Chern numbers.

THEOREM A. Let $u_{M}$ be a homogeneous polynomial in the Chern classes of a weakly complex manifold $M^{n}$ such that any n-dimensional homogenous polynomial $w_{M}$ with $u_{M}$ as a factor (i.e., $w_{M}=u_{M} \cdot v_{M}$ ) has value zero on the basic class of $M$. Then $M$ is complex cobordant to a manifold $M^{\prime}$ with $u_{M^{\prime}}$ of finite order.

Further, if the normal bundle of $M$ is trivial over its $(2 q-1)$-skeleton, the same may be assumed for $M^{\prime}$.

Let $X$ be an $n$-dimensional complex cobordism class, and let $K(X)$ $=\bigcup_{M \in X} \operatorname{Ker} f_{M}^{*}$, where $f_{M}: M \rightarrow B U$ is a classifying map for the (stable) normal bundle, and $f_{M}^{*}: H^{*}(B U ; Q) \rightarrow H^{*}(M ; Q)$ is the induced homomorphism in the rational coefficients. (For $M$ empty, $\operatorname{Ker} f_{M}^{*}=H^{*}(B U ; Q)$.) Then Theorem A implies $K(X)$ is an ideal. Let $C(X)=H^{*}(B U ; Q) / K(X)$; it is a graded algebra called the rational characteristic ring of $X$.

THEOREM B (PoINCARÉ DUALITY FOR COMPLEX COBORDISM ClASSES). The map $C^{i}(X) \otimes C^{n-1}(X) \rightarrow C^{n}(X)$ induced by cup product gives a pairing of $C^{i}(X)$ and $C^{n-i}(X)$ to $Q$; the isomorphism $C^{n}(X) \simeq Q(X \neq 0)$ being given by associating to each monomial in $\mathrm{H}^{n}(B U ; Z)$ the corresponding Chern number, extending linearly, and then passing to the quotient. (If $X=0$, then all the groups $C^{i}(X)$ are zero.)

Theorem $\mathrm{B}$ is an immediate consequence of Theorem A.

Received by the editors September 27, 1962. 
In order to prove these theorems we first generalize cobordism theory to arbitrary (not necessarily differentiable) topological groups with specified homomorphism into the orthogonal group; or equivalently, arbitrary spaces with specified homotopy class of maps into the universal base space of the orthogonal group. Given a connected countable CW-complex $C$ and a map $f: C \rightarrow B O_{h}$, we may assume that $f$ is a bundle projection of an associated bundle to the universal $O_{k}$-bundle (i.e., there exists a homotopy equivalence of the total space with $C$ and the projection corresponds to $f$ under this equivalence). If a smooth manifold $M^{n}$ is immersed in $E^{n+k}$ (Euclidean $n+k$-space), we will say that its normal bundle has a $(C, f)$-structure if we are given a specific homotopy class of lifts $\tilde{v}$ of the normal map, $v: M^{n} \rightarrow G_{k n} \subset B O_{k}$, to $C$; i.e., $v=f \tilde{v}$. Now given a sequence of pairs $\left(C_{k}, f_{k}\right), f_{k}: C_{k} \rightarrow B O_{k}$, and maps $j_{k}: C_{k} \rightarrow C_{k+1}$ such that $i_{k} f_{k}=f_{k+1} j_{k}$, where $i_{k}: B O_{k} \rightarrow B O_{k+1}$ is induced by the inclusion $O_{k} \subset O_{k+1}$; we will say $M^{n}$ has a $(C, f)$-structure, $(C, f)=\left\{\left(C_{k}, f_{k}\right)\right\}$, if the normal bundle of $M^{n}$ in some $E^{n+k}$ has a $\left(C_{k}, f_{k}\right)$-structure. By considering $M^{n} \subset E^{n+k} \subset E^{n+k+i}$, it is clear that a $\left(C_{k}, f_{k}\right)$-structure defines a unique $\left(C_{k+r}, f_{k+r}\right)$-structure, $r=0,1,2, \cdots$, and we identify these structures. Further, given two immersions of $M^{n}$ in $E^{n+k}, k$ sufficiently large, they are regularly homotopic, and any two such regular homotopies are themselves homotopic through regular homotopies. By using the covering homotopy theorem on $f_{k}: C_{k} \rightarrow B O_{k}$, we get a 1-1 correspondence between $(C, f)$-structures defined by the two immersions. This enables us to define a $(C, f)$-structure on $M^{n}$ independent of the particular immersion. A $(C, f)$ cobordism theory may then be defined in the usual way. Letting $\gamma_{k}$ be the universal $k$-dim vector bundle over $B O_{k}$, and $T\left(f_{k}^{*}\left(\gamma_{k}\right)\right)$. The Thom space of the induced vector bundle over $C_{k}$, we prove:

THEOREM C. The $(C, f)$-cobordism group of closed n-manifolds is isomorphic to $\operatorname{Lim} \pi_{n+k}\left(T f_{k}^{*}\left(\gamma_{k}\right)\right)$.

Our general technique is also used to obtain the following results:

THEOREM D. Let $M^{n}$ be a closed weakly complex manifold with $c_{1}=c_{2}$ $=\cdots=c_{k}=0,2 k<n / 2$; then $M$ is complex cobordant to a $2 k$-connected manifold.

THEOREM $\mathrm{D}^{\prime}$. Let $M^{4 s}, s>1$, be a closed weakly complex manifold with $c_{1}=c_{2}=\cdots=c_{s-1}=0$, and $c_{s}^{2}=0$; then $M$ is complex cobordant to an almost parallelizable manifold.

THEOREM E. In every homotopy class of maps of $S^{m+4 k} \rightarrow S^{m}, m>4 k+1$, there exists a differentiable map $f$ such that $f^{-1}$ (base point of $S^{m}$ ) $=M^{4 k}$, where $M^{4 k}$ is a smooth manifold homeomorphic to a sphere $S^{4 k}$ and imbedded in $S^{m+4 k}$ with a trivial normal bundle.

Theorem $\mathrm{E}$ has obvious consequences for differentiable structures on $4 k$-di- 
mensional spheres, but Kervaire and Milnor [16] have much more general results in this direction.

The author wishes to thank R. F. Williams and E. Dyer for many helpful conversations. In particular, Theorem $\mathrm{E}$ is a joint result of $\mathrm{E}$. Dyer and the author. Also, the referee has made a number of helpful suggestions.

1. Lifting the group of a bundle. Given a homomorphism of topological groups $\rho: G \rightarrow H$, we may ask when an $H$-bundle(1) may be lifted to $G$; i.e., is induced from a $G$-bundle by $\rho$. For the case that $\rho$ is an inclusion, a simple criterion is given in [13]. We generalize this result as follows: Let $\left(E_{G}, G, B_{G}\right)$ be a universal bundle; i.e., $\pi_{i}\left(E_{G}\right)=0$ all $i$ (such bundles always exist [6]), and let $G$ act on $E_{G} \times H$ by right action in $E_{G}$ and right translation on $H$ (via $\rho$ ). Let $H$ act on $E_{G} \times H$ by left translation on the second factor and trivial action on the first. Since the actions of $H$ and $G$ commute, we get a well-defined action of $H$ on $\left(E_{G} \times H\right) / G$.

Proposition 1.1. Given $\rho: G \rightarrow H$, an $H$-bundle can be lifted to $G$ if and only if the associated bundle with fibre $F=\left(E_{G} \times H\right) / G$ has a cross-section.

Proof. Let $\left(E_{H}, H, B_{H}\right)$ be a universal bundle for $H$. Let $\widetilde{B}_{G}=\left(E_{G} \times E_{H}\right) / G$, $G$ acting on the right of $E_{G}$ and on the right of $E_{H}$ via $\rho$. Then $\left(E_{G} \times E_{H}, G, \widetilde{B}_{G}\right)$ is again a universal bundle for $G$. On the other hand, defining $\tilde{f}: \widetilde{B}_{G} \rightarrow B_{H}$ by passage to the quotients from $E_{G} \times E_{H} \stackrel{p_{2}}{\rightarrow} E_{H}$, we see that $\widetilde{B}_{G}$ is a bundle over $B_{H}$ with fibre $F$, associated to $\left(E_{H}, H, B_{H}\right)$. Further, since $p_{2}((x, y) g)=p_{2}(x g, y \rho(g))$ $=y \rho(g), x \in E_{G}, y \in E_{H}, g \in G ; \tilde{f}^{*}\left(E_{H}, H, B_{H}\right) \simeq \rho_{*}\left(E_{G} \times E_{H}, G, \widetilde{B}_{G}\right)$. Hence for a bundle with group $H$, the following are equivalent:

(1) The bundle is induced from a $G$-bundle via $\rho$.

(2) The classifying map into $B_{H}$ may be lifted to $\widetilde{B}_{G}$.

(3) The associated bundle with fibre $\left(E_{G} \times H\right) / G$ has a cross-section.

Let $W_{0}$ be the category of spaces of the same homotopy type as a countable $\mathrm{CW}$-complex. Looking at Proposition 1.1 from the point of view of the universal base spaces we get:

Proposition 1.2. Let $B \in W_{0}$ be connected, and let $\mu_{H}=\left(E_{H}, H, B_{H}\right)$ be any universal bundle. Then:

1. Any map $f: B \rightarrow B_{H}$ may be replaced by a bundle projection. Explicitly, there is a weak homotopy equivalence $\psi: B \rightarrow \widetilde{B}$; and a bundle projection $\tilde{f}: \widetilde{B} \rightarrow B_{H}$ of a bundle $\left(\widetilde{B}, F, B_{H}\right)$ with fibre $F \simeq$ the total space of $f^{*} \mu_{H}$ (associated to $\left.\mu_{H}\right)$; such that $\tilde{f} \psi$ is homotopic to $f$. Further there is a map $\pi: \widetilde{B} \rightarrow B$ such that $\pi \psi$ is homotopic to the identity; and if $E_{H}$ is contractible, $\psi$ is a homotopy equivalence with inverse $\pi$.

(1) Al! bundles are locally trivial. 
2. If $g: X \rightarrow B_{H}$ is a classifying map of an $H$-bundle over $X$, the homotopy classes of cross-sections of the associated bundle with fibre $F$ are in 1-1 correspondence with the homotopy classes of maps of $X$ into $\widetilde{B}$ covering $g$ (the homotopies being covering maps at each stage).

Corollary 1.3 (Fadell). Any map $f: B \rightarrow C, B, C \in W_{0}$ with $B$ and $C$ connected, may be replaced by a bundle projection $\tilde{f}: \widetilde{B} \rightarrow \widetilde{C}$; i.e., there exist homotopy equivalences $\psi: B \rightarrow \widetilde{B}$ and $\lambda: \widetilde{C} \rightarrow C$ such that $\lambda \tilde{f} \psi$ is homotopic to $f$.

Proof of Proposition 1.2. We may assume $B$ is a countable connected simplicial complex in the weak topology [7]. Then Milnor [5] has shown that there exists a contractible universal bundle $\left(E_{G}, G, B_{G}\right), B_{G}=B, E_{G}$ and $G C W$-complexes, such that for any principal bundle $\xi_{H}$ over $B$ (with group $H$ ) there exists a homomorphism $\rho: G \rightarrow H$ such that $\xi_{H} \simeq \rho_{*}\left(E_{G}, G, B_{G}\right)$.

Choose $\rho: G \rightarrow H$ so that $\rho_{*}\left(E_{G}, G, B_{G}\right) \simeq f^{*} \mu_{H}$. Now apply Proposition 1.1, and let $\widetilde{B}=\widetilde{B}_{G}$ as defined in the proof of that proposition. Then (2) of 1.2 follows immediately. Now the $F$ of 1.1 is essentially the total space of $\rho_{*}\left(E_{G}, G, B_{G}\right)$, (we have switched the action of $G$ and $H$ to the opposite sides of $H$, but these correspond under the inverse map) and hence is isomorphic to the total space of $f^{*} \mu_{H}$. It remains to define $\psi$ and $\pi$.

According to the definition of $\rho$, there exists a map $\theta: E_{G} \rightarrow E_{H}$ satisfying $\theta(x g)=\theta(x) \rho(g), x \in E_{G}, g \in G$ and covering $f$. Define $\psi: B=B_{G} \rightarrow \widetilde{B}_{G}$ by passage to the quotient from the map $x \rightarrow(x, \theta(x))$ of $E_{G} \rightarrow E_{G} \times E_{H}$. Then $\tilde{f} \psi=f$. Now $\widetilde{B}_{G}=\left(E_{G} \times E_{H}\right) / G$ is a bundle over $B$ with projection map $\pi: \widetilde{B}_{G} \rightarrow B$ defined by passage to the quotient from $p_{1}: E_{G} \times E_{H} \rightarrow E_{G}$. Then $\pi \psi=1$ and the fibre of $\pi$ is $E_{H}$. Since $\pi_{i}\left(E_{H}\right)=0$ for all $i$, it follows that $\pi$ and $\psi$ are both weak homotopy equivalences. Finally, if $E_{H}$ is contractible, the bundle $\left(\widetilde{B}_{G}, E_{H}, B, \pi\right)$ is the same fibre homotopy type as the trivial bundle over $B$ with fibre a single point. (See [2].) This implies in particular that $\psi \pi$ is homotopic to the identity.

Proof of corollary. We may assume that $B$ and $C$ are both countable connected locally finite simplicial complexes. Then as in the proof above $C$ is the base space of a contractible universal bundle. The corollary now follows from the proposition.

Definition. Let $\left(B, F, B O_{n}\right)$ be any associated bundle to the universal bundle for $O_{n}$, and let $f: B \rightarrow B O_{n}$ be the bundle projection. We will say that an $n$-plane bundle $\xi$ over a space $X$ is $(B, f)$-reducible if the associated bundle over $X$ with fibre $F$ has a cross-section. A $(B, f)$-structure on $\xi$ is an isotopy class of crosssections of the associated bundle with fibre $F$ (i.e., a homotopy class of crosssections, where the homotopies are cross-sections at each stage).

If instead of a bundle we are given only a map $f: B \rightarrow B O_{n}$ of a space $B \in W_{0}$ into the universal bundle for $O_{n}$. We will say that an $n$-plane bundle $\xi$ over a paracompact space $X$ is $(B, f)$-reducible if it is $(\widetilde{B}, \tilde{f})$-reducible; i.e., if the associated 
bundle to $\xi$ over $X$ with fibre $F=f^{*} E O_{n}$ has a cross-section. A $(B, f)$-structure on $\xi$ is an isotopy class of cross-sections of the associated bundle with fibre $F$.

Explicitly, if $P(\xi)$ is the principal bundle of $n$-frames corresponding to $\xi$, then the associated bundle with fibre $F$ is $\left(\left(P(\xi) \times f^{*} E O_{n}\right) / O_{n}, f^{*} E O^{n}, O_{n}, X\right)$.

REMARK. The property of $(B, f)$-reducibility depends only on the equivalence class of the bundle $\xi$. A $(B, f)$-structure on the other hand, is for a specific bundle, and there is no way in general of corresponding $(B, f)$-structures for different but equivalent bundles.

Starting with a bundle map of the $n$-plane bundle $\xi$ into the universal $n$-plane bundle, one gets a uniquely defined map of the principle bundles, and hence of the associated bundle $(P(\xi) \times F) / O_{n}$ into $\left(E O_{n} \times F\right) / O_{n}$. If $g: X \rightarrow B O_{n}$ is the map on base spaces under the above bundle maps, then any lifting $g: X \rightarrow\left(E O_{n} \times F\right) / O_{n}$ defines a unique cross-section of the associated bundle to $\xi$ and conversely. Note that this correspondence depends in general on the explicit bundle map of the associated bundles.

2. Thom spaces and cobordism. Let $G_{r, n}$ be the Grossmann manifold of (unoriented) $r$-planes in $E^{r+n}$. Let $\gamma_{n}^{r}$ be the $r$-plane bundle over $G_{r, n}$ consisting of pairs: an $r$-plane together with a vector in the $r$-plane. Then $B O_{r}=\operatorname{Lim}_{n} G_{r, n}$, and the universal $r$-plane bundle $\gamma^{r}=\operatorname{Lim} \gamma_{n}^{r}$.

Let $M^{n}$ be a smooth $\left(C^{\infty}\right)$ compact manifold with or without boundary. An imbedding $i: M \rightarrow E^{n+r}$ gives a naturally defined map of the bundle of vectors normal to $M$ into $\gamma_{n}^{r}$, by translating a normal vector at a point $x \in M$, together with the normal plane to $M$ at $x$, to the origin of $E^{n+r}$. This, followed by the inclusion of $\gamma_{n}^{r}$ into $\gamma^{r}$, gives a bund!e map of the normal bundle to $i(M)$ into the universal $r$-plane bundle. Let $v(i): M \rightarrow B O_{r}$ be the induced map on base spaces.

If $\left(B, F, B O_{r}, f\right)$ is any associated bundle to the universal $O_{r}$-bundle, we will say that $i: M \rightarrow E^{n+r}$ has a $(B, f)$-structure, if we are given an isotopy class of liftings $\tilde{v}(i): M \rightarrow B, f \tilde{v}(i)=v(i)$. (See discussion at the end of $\$ 1$.)

If $r$ is sufficiently large ( $r$ depends on $n$ only), any two imbeddings $i_{1}, i_{2}: M^{n} \rightarrow E^{n+r}$ are regularly homotopic, and any two such regular homotopies are themselves homotopic through regular homotopies leaving endpoints fixed [3]. This enables us to prove:

LEMMA 2.1. If $r$ is sufficiently large, then there is a one-to-one correspondence between the $(B, f)$-structures on $i_{1}, i_{2}: M \rightarrow E^{n+r}, i_{1}$ and $i_{2}$ any imbeddings. Consequently, we may speak of a $(B, f)$-structure on $M^{n}$ independent of the particular imbedding.

Proof. Let $\tilde{v}\left(i_{1}\right): M \rightarrow B, f \tilde{v}\left(i_{1}\right)=v\left(i_{1}\right)$. Now a regular homotopy from $i_{1}$ to $i_{2}$ gives a homotopy between $v\left(i_{1}\right)$ and $v\left(i_{2}\right)$ and any two such homotopies are 
homotopic with end points fixed. Using the covering homotopy theorem in $\left(B, F, B O_{r}, f\right)$ we get a homotopy of $\tilde{v}\left(i_{1}\right)$ to a map $\tilde{v}\left(i_{2}\right): M \rightarrow B$ covering $v\left(i_{2}\right)$. We claim the class of $\tilde{v}\left(i_{2}\right)$ depends only on the class of $\tilde{v}\left(i_{1}\right)$.

Let $\tilde{v}^{\prime}\left(i_{1}\right)$ be any other lift of $v\left(i_{1}\right)$ in the same class and $\tilde{v}^{\prime}\left(i_{2}\right)$ be the lift of $v\left(i_{2}\right)$ obtained from $\tilde{v}^{\prime}\left(i_{1}\right)$ by any covering homotopy. This defines a map $\tilde{\psi}$ of $M \times T$ into $B$, where $T$ is the subspace of $I \times I$ consisting of three edges of the square. Further $\tilde{\psi}$ covers a map $\psi: M \times T \rightarrow B O_{r}$ with $\psi\left(x, t_{1}, 0\right)=v\left(i_{1}\right)(x)$, and $\psi\left(x, 0, t_{2}\right), \psi\left(x, 1, t_{2}\right)$ being two homotopies between $v\left(i_{1}\right)$ and $v\left(i_{2}\right)$ which are themselves homotopic with end points fixed. Thus $\psi$ is extendable to $M \times I \times I$ with $\psi\left(x, t_{1}, 1\right)=v\left(i_{2}\right)$. Consequently, $\tilde{\psi}$ is extendable to $M \times I \times I$ with $\tilde{\psi}\left(x, t_{1}, 1\right)$ over $v\left(i_{2}\right)$ and giving the desired isotopy between $\tilde{v}\left(i_{2}\right)$ and $\tilde{v}^{\prime}\left(i_{2}\right)$. This establishes the above claim.

Now applying the same argument, a class of lifts $\tilde{v}\left(i_{2}\right)$ of $v\left(i_{2}\right)$ defines a unique class of lifts of $v\left(i_{1}\right)$; and by a further application of the same argument the correspondence $\tilde{v}\left(i_{1}\right)$ to $\tilde{v}\left(i_{2}\right)$ to $\tilde{v}^{\prime}\left(i_{1}\right)$ returns us to the same class. This establishes the lemma.

Now suppose we are given a sequence $(B, f)$ of bundle projections $f_{r}: B_{r} \rightarrow B O_{r}$ (associated to the universal bundle) and maps $g_{r}: B_{r} \rightarrow B_{r+1}$ such that

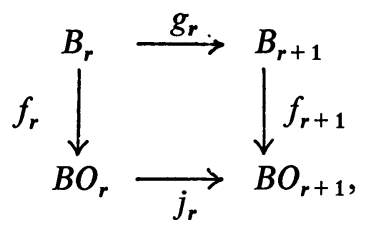

where $j_{r}$ is the standard inclusion. Then a $\left(B_{r}, f_{r}\right)$-structure on $M^{n}$ in $E^{n+r}$ defines a unique $\left(B_{r+1}, f_{r+1}\right)$-structure on $M^{n} \subset E^{n+r} \subset E^{n+r+1}$. This sequence of structures denoted $\xi=\left(\xi_{r}\right)$ will be called a $(B, f)$-structure on $M^{n}$, and $\left(M^{n}, \xi\right)$ a $(B, f)$-manifold. Two such structures will be identified if they correspond for some $r$ (and hence for all larger $r$ ) under the covering homotopy theorem. The above lemma then shows that this structure is well defined.

If instead of bundles, we are given only spaces $C_{r}$, and maps $f_{r}: C_{r} \rightarrow B O_{r}$, and $g_{r}: C_{r} \rightarrow C_{r+1}$, such that $f_{r+1} g_{r}$ and $j_{r} f_{r}$ are the same up to homotopy; we may replace the pairs $\left(C_{r}, f_{r}\right)$ by equivalent bundle pairs according to $\S 1$, at least if $C_{r} \in W_{0}$. The above diagram will then commute up to homotopy, but by using the covering homotopy theorem, we may inductively deform the maps $g_{r}$ so that the above diagram commutes. As we will see later, the cobordism groups we are about to define depend only on the homotopy type of the pairs $\left(C_{r}, f_{r}\right)$ and maps $g_{r}: C_{r} \rightarrow C_{r+1}$.

Let $M_{i}^{n}, \quad i=1,2$, be two closed manifolds. Let $\left(W^{n+1}, \xi\right)$ be a $(B, f)$-manifold with boundary the disjoint union of $M_{1}$ and $M_{2}$. We will define induced $(B, f)$-structures on $M_{i}$. By standard imbedding theorems [8] one may imbed 
$W^{n+1}$ in $E^{n+r+1}, r$ sufficiently large, so that $W^{n+1}$ lies between two parallel $(n+r)$-planes $E_{i}^{n+r}, i=1,2$, with its boundaries $M_{i}$ imbedded in $E_{i}^{n+r}$ and $W^{n+1}$ meeting $E_{i}^{n+r}$ perpendicularly. We take a fixed orientation on our Euclidean spaces and take $E_{2}^{n+r}$ on the positive side of $E_{1}^{n+r}$. Then the normal planes to $M_{i}$ in $E_{i}^{n+r}$ are just the restriction to $M_{i}$ of the normal planes to $W$ in $E^{n+r+1}$. If $v: W \rightarrow B O_{r}$ is the normal map, and the $(B, f)$-structure on $W$ is represented by an isotopy class of liftings $\tilde{v}: W \rightarrow B_{r}$; then $\tilde{v} \mid M_{i}$ will be a lifting of $v \mid M_{i}$ and define a $\left(B_{r}, f_{r}\right)$-structure on $M_{i}$ in $E^{n+r}$. Using the same arguments as in Lemma 2.1 above, it is easy to check that the $(B, f)$-structures on $M_{i}$ so defined are independent of the particular imbedding of the above type of $W^{n+1}$.

We can now make the

Definition. Let $\left(M_{i}^{n}, \xi^{i}\right), i=1,2$, be two closed $(B, f)$-manifolds. We will say they are $(B, f)$-cobordant if there exists a $(B, f)$ manifold $\left(W^{n+1}, \xi\right)$ such that:

(a) $\partial W^{n+1}$ is the disjoint union of $M_{1}$ and $M_{2}$.

(b) The $(B, f)$-structures on $M_{1}$ and $M_{2}$ induced by that on $W$ coincide with $\xi_{1}$ and $\xi_{2}$, respectively.

LEMMA 2.2. ( $B, f)$-cobordism is an equivalence relation, and the cobordism classes of closed $(B, f)$-manifolds form an abelian group under addition induced by disjoint union of $(B, f)$-manifolds.

Proof. We leave most of the proof to the reader. See for example [8]. We will consider only the construction of an inverse. Given a $(B, f)$-manifold $\left(M^{n}, \xi\right)$, imbed $M^{n}$ in $E^{n+r}, r$ sufficiently large, and imbed $M^{n} \times I$ in $E^{n+r} \times E$ $=E^{n+r+1}$. The normal map $v: M^{n} \times I \rightarrow B O_{r}$ is simply the projection of $M^{n} \times I$ onto $M^{n}$ followed by the normal map of $M^{n}$, and hence the $(B, f)$-structure on $M^{n}$ defines a $(B, f)$-structure $\xi^{\prime}$ on $W^{n+1}=M^{n} \times I$. Now deform $W$ through a regular homotopy so that $W$ is imbedded in the interior of $E^{n+r} \times I$, except for $M^{n} \times O$, and $M^{n} \times 1$ which are imbedded disjointly in $E^{n+r} \times O$, and $W$ meets $E^{n+r} \times O$ perpendicularly. This induces a structure $\xi^{\prime}$ on $M^{n} \times 1$, and by the definition of class addition $\left[M^{n}, \xi\right]+\left[M^{n}, \xi^{\prime}\right]=0$. It is clear that $\xi^{\prime}$ depends only on $\xi$, and we write $-\xi$ for $\xi^{\prime}$. (In the case that $B_{r}=B S O_{r}$ and $f_{r}$ is the standard covering map, $\xi$ is just an orientation of $M^{n}$ and $-\xi$ is the opposite orientation.)

It is possible to define $(B, f)$-structures and $(B, f)$-cobordism in terms of the stable tangent bundle as follows: Let $B=\operatorname{Lim}\left(B_{r}, g_{r}\right), f=\operatorname{Lim} f_{r}: B \rightarrow B O$. Let $I: B O \rightarrow B O, B O=B O_{\infty}$, be the map induced from $I_{n, N}: G_{n, N} \rightarrow G_{N, n}\left(I_{n, N}\right.$ sends an $n$-plane into its orthogonal $N$-plane) by passage to the limit. Note that $I^{2}=$ identity. Then $B$ is a bundle over $B O$ with fibre $F=\operatorname{Lim} F_{r}$, and $I^{*} B$ is also such a bundle over $B O$. Since $I^{*} E O=E O, I^{*} B=B$. Let $\tau(M)$ be the tangent bundle of $M$, and let $P(\tau)$ be the associated principal bundle with group $O$, and consider the associated bundle $\left.\tau_{f}=(P(\tau) \times F) / O, F, O, M\right)$ with fibre $F$. We define a $(B, f)$-structure $\xi$ on $M$ as an isotopy class of cross-sections of $\tau_{f}$. Two closed 
$(B, f)$-manifolds $\left(M_{1}^{n}, \xi_{1}\right),\left(M_{2}^{n}, \xi_{2}\right)$ will be called $(B, f)$-cobordant if there exists a $(B, f)$-manifold $\left(W^{n+1}, \xi\right)$, with boundary the disjoint union of $M_{1}, M_{2}$, with $\xi \mid M_{i}=\xi_{i}$.

To show the correspondence between the definitions of $(B, f)$-manifolds in terms of tangent and normal bundles, let $M^{n}$ be imbedded in $E^{n+N}, N$ sufficiently large. The map $v_{N}: M^{n} \rightarrow G_{N, n}$ and $\tau_{N}: M^{n} \rightarrow G_{n, N}$ are related by $\tau_{N}=I_{N, n} v_{N}$. Let $v: M^{n} \rightarrow B O$, and $\tau: M^{n} \rightarrow B O$ be the maps obtained by passage to the limit, then $\tau=I v$. Further, these are covered by explicit bundle maps $N: v_{f} \rightarrow B$, $T: \tau_{f} \rightarrow B$ of the associated bundles with fibre $F$, such that $T=I N$ (denoting again by $I$ the bundle map of $B$ into $B$ covering $I: B O \rightarrow B O)$. A $(B, f)$-structure $\xi_{N}$ on $M$ defined in terms of the normal bundle corresponds via $N$ to an isotopy class of lifts $\tilde{v}$ of $v$ to $B$. Then $I \tilde{v}$ gives an isotopy class of lifts of $\tau$ to $B$, and hence via $T$ to a $(B, f)$-structure $\xi_{T}=I \xi_{N}$ in terms of the tangent bundle. Conversely, starting with $\xi_{T}$ we may define in the same way a $(B, f)$-structure $\xi_{N}=I \xi_{T}$. Since $I^{2}$ is the identity, this establishes a $1-1$ correspondence between $(B, f)$-structures defined in terms of tangent and normal bundles. By Lemma 2.1, this correspondence is independent of the imbedding, if $N$ is sufficiently large. It now follows that this correspondence also defines a 1-1 correspondence between $(B, f)$-cobordism classes defined in terms of tangent and normal bundles, respectively.

Considering $B O_{r}$ to be the space of $r$-planes contained in some $E^{s} \subset E^{\infty}$, and defining the usual metric on the subspace of $E^{\infty}$ consisting of vectors with only a finite number of coordinates nonzero, we obtain a Riemannian metric on the universal vector bundle $\gamma^{r}$ over $B O_{r}$. Consequently, we may form the Thom space $T B O_{r}$ of $\gamma^{r}$ by identifying all vectors of length $\geqq 1$ to a single point denoted $\infty$. Since $B O_{r}=\lim G_{r, m}$, it is easy to see that $T B O_{r}=\lim T G_{r, m}$. Now define Thom space of $\left(B_{r}, f_{r}\right), T\left(B_{r}, f_{r}\right)$ (or simply $T B_{r}$ if no confusion arises) as the Thom space of the $r$-plane bundle $f_{r}^{*}\left(\gamma^{r}\right)$ (with the induced metric). Write $T f_{r}: T B_{r} \rightarrow O_{r}$ for the naturally induced map. Now $j_{r}^{*}\left(\gamma^{r+1}\right)$ is also a vector bundle over $B O_{r}$, isomorphic to the Whitney sum of $\gamma^{r}$ and a trivial line bundle; hence the Thom space of $j_{r}^{*}\left(\gamma^{r+1}\right)$ may be identified to the suspension $\Sigma T B O_{r}$ of the Thom space of $\gamma_{r}$. It is easy to see then that the commutative diagram above leads to the commutative diagram:

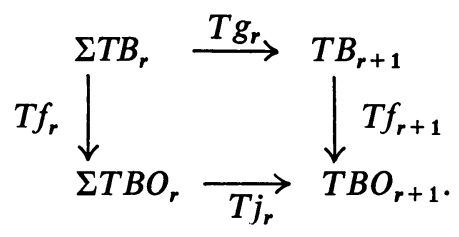

In particular, $T g_{r} \circ \Sigma$ induces a homomorphism $\pi_{n+r}\left(T B_{r}, \infty\right) \rightarrow \pi_{n+r+1}\left(T B_{r+1}, \infty\right)$.

THEOREM. The cobordism group $X^{n}$ of $n$-dimensional $(B, f)$-manifolds is isomorphic to $\operatorname{Lim}_{r} \pi_{n+r}\left(T f_{r}^{*}\left(\gamma^{r}\right)\right)$. 


\section{Proof.}

(a) Definition of $\Theta: X^{n} \rightarrow \operatorname{Lim} \pi_{n+r}\left(T f_{r}^{*}\left(\gamma^{r}\right)\right)$.

Let $M^{n} \subset E^{n+r}$ with a $\left(B_{r}, f_{r}\right)$-structure, i.e., we are given a map $\tilde{v}: M^{n} \rightarrow B_{r}$ such that $f_{r} \tilde{v}=v: M^{n} \rightarrow B O_{r}$. Now $v$ is covered by a bundle map $n: N \rightarrow \gamma^{r}$ of the normal bundle of $M$ in $E^{n+r}$ by translating normal vectors to the origin. The exponential map exp :N $\rightarrow E^{n+r}$ is an embedding for the subspace $N_{\varepsilon}$ of vectors of length less than or equal to $\varepsilon$, for $\varepsilon$ sufficiently small. By changing scale if necessary, we may assume that $n$ takes $N_{\varepsilon}$ onto vectors of length less than or equal to one in $\gamma^{r}$. We define a map $\theta^{\prime}: S^{n+r}=E^{n+r} \cup p \rightarrow T\left(\gamma^{r}\right)$ by sending all points of $S^{n+r}$ outside or on the boundary of $N_{\varepsilon}$ to $\infty$. The lift $\tilde{v}$ together with $\theta^{\prime}$ then defines a unique map $\theta: S^{n+r} \rightarrow T\left(f_{r}^{*}\left(\gamma^{r}\right)\right)$ with $T f_{r} \theta=\theta^{\prime}$. Clearly the map obtained by considering $M^{n} \subset E^{n+r} \subset E^{n+r+1}$ is just $T g_{r} \circ \Sigma \theta$. Thus we get an element of $\operatorname{Lim} \pi_{n+r}\left(T f_{r}^{*}\left(\gamma^{r}\right)\right)$.

To show that this element depends only on the cobordism class of $M^{n}$ (and is independent of the imbedding) let $M_{1}^{n}, M_{2}^{n}$ be cobordant $(B, f)$-manifolds; i.e., there exists a $(B, f)$-manifold $W^{n+1}$ with $\partial W^{n+1}$ the disjoint union of $M_{1}$ and $M_{2}$ and inducing the given $(B, f)$-structures on $M_{1}$ and $M_{2}$. This means we may imbed $W^{n+1}$ in $E^{n+r+1}, r$ sufficiently large, so that the imbedding of $\partial W^{n+1}$ coincides with given imbeddings of $M_{i}$ in parallel $E_{i}^{n+r}, i=1,2$. Further the lift $\tilde{v}: W \rightarrow B_{r}$ may be assumed to coincide with the lifts $\tilde{v}_{i}: M_{i} \rightarrow B_{r}$ when restricted to $\partial W$. Define the bundle map $n$ of $N_{\varepsilon} \subset E^{n+r} \times I$ onto the vectors of length less than or equal to one of $\gamma^{r}$, and the maps $\theta^{\prime}:\left(E^{n+r} \cup p\right) \times I \rightarrow T\left(\gamma^{r}\right)$ and $\theta: S^{n+r} \times I \rightarrow T f_{r}^{*}\left(\gamma^{r}\right)$ as above. As the homotopy class of $\theta_{i}$ is unaffected by taking smaller $\varepsilon$ 's if necessary, we may assume all maps are defined for the same $\varepsilon$. Then $\theta \mid S^{n+r} \times 0$ and $\theta \mid S^{n+r} \times 1$ coincides with $\theta$ and $\theta_{2}$, respectively, and hence represent the same homotopy class in $\pi_{n+r}\left(T f_{r}^{*}\left(\gamma^{r}\right)\right)$. Thus we get a well-defined map $\Theta: X^{n} \rightarrow \operatorname{Lim}_{r} \pi_{n+r}\left(T f_{r}^{*}\left(\gamma^{r}\right)\right)$.

(b) $\Theta$ is a homomorphism.

Let $M_{1}$ and $M_{2}$ represent two cobordism classes of $X^{n}$, and imbed them on different sides of $E^{n+r-1}$ in $E^{n+r}, r$ sufficiently large. Then $\Theta\left(\left[M_{1}\right]+\left[M_{2}\right]\right)$ is represented by a map $\theta=\theta_{1} \vee \theta_{2}$, where $\theta_{i}: S^{n+r} \rightarrow T f_{r}^{*}\left(\gamma^{r}\right)$ represent $\left[M_{i}\right]$; i.e., $\Theta\left(\left[M_{1}\right]+\left[M_{2}\right]\right)=\Theta\left(\left[M_{1}\right]\right)+\Theta\left(\left[M_{2}\right]\right)$.

(c) $\Theta$ is onto.

Let $\theta:\left(S^{n+r}, p\right) \rightarrow\left(T f_{r}^{*}\left(\gamma^{r}\right), \infty\right), r$ sufficiently large, then $T f_{r}^{\circ} \theta:\left(S^{n+r}, p\right) \rightarrow\left(T\left(\gamma^{r}\right), \infty\right)$. Since $T\left(\gamma^{r}\right)=T B O_{r}=\operatorname{Lim} T G_{r, s}$, and $S^{n+r}$ is compact, $T f_{r} \circ \theta\left(S^{n+r}\right) \subset T G_{r, s}$, for some s. Using standard techniques [8] or [14], $T f_{r} \circ \theta$ may be deformed to a map $h_{r}$ so that:

(1) $h_{r}$ is differentiable in the preimage of some open neighborhood of $G_{r, s} \subset T G_{r s}$, and $t$-regular on $G_{r, s}$.

(2) If $M^{n}=h_{r}^{-1}\left(G_{r . s}\right), h_{r}$ is a bundle map on a normal tube of $M^{n}$ in $E^{n+r}$ $=S^{n+r}-p$. 
(3) The map is left unchanged on the preimage $V$ of some closed neighborhood of $\infty$.

Since $h_{r} \mid M$ is a classifying map for the normal bundle of $M$, we may assume (by a further deformation if necessary) that:

(4) $h_{r} \mid M$ is the normal map $v: M \rightarrow G_{r, n} \subset G_{r, s}$, and $h_{r}$ on some normal tube of $M$ is just translation of vectors to the origin. Now notice that $T f_{r}: T B_{r} \rightarrow T B O_{r}$ is a bundle map except at the point $\infty$. Let $V_{0}$ be the interior of $V$. Then $T f_{r} \circ \theta\left(S^{n+r}-V_{0}\right)$ does not contain $\infty$, and so the covering homotopy theorem holds, and the deformation of $T f_{r} \circ \theta$ on $S^{n+r}-V_{0}$ may be covered by a deformation of $\theta$ on $S^{n+r}-V_{0}$, which is stationary on the boundary of $V$. Hence we may cover the deformation of $T f_{r} \circ \theta$ by one of $\theta$ to $\theta_{1}$. Then $\theta_{1}^{-1}\left(B_{r}\right)=\left(T f_{r} \circ \theta_{1}^{-1}\right)\left(G_{r, s}\right)=h_{r}^{-1}\left(G_{r, s}\right)=M^{n}$. Further $\theta_{1} \mid M: M \rightarrow B_{r}$ is a lift of $h_{r} \mid M=v$. This defines a $\left(B_{r}, f_{r}\right)$-structure on $M^{n}$ in $E^{n+r}$ and hence a $(B, f)$ structure on $M^{n}$. On the other hand, defining $\Theta([M])$ using the above imbedding of $M^{n}$ in $E^{n+r}, \Theta=\left(\theta_{1}\right)$, i.e., $\Theta([M])$ is the given element of $\operatorname{Lim} \pi_{n+r}\left(T f_{r}^{*}\left(\gamma^{r}\right)\right)$ and $\Theta$ is onto.

(d) $\Theta$ is one-to-one.

Let $M$ be a $(B, f)$-manifold such that $\Theta([M])=0$; we must show that $M$ is $(B, f)$-cobordant to 0 . Now $\Theta([M])$ is represented by a map $\theta_{1}: S^{n+r} \rightarrow T B_{r}$ which is homotopic to $\theta_{0}: S^{n+r} \rightarrow \infty$ by a homotopy $L: S^{n+r} \times I \rightarrow T B_{r}$. Then $T f_{r} \circ \theta_{1}$ is homotopic to $T f_{r} \circ \theta_{0}$ by $T f_{r} \circ L$. Again $T f_{r} \circ L\left(S^{n+r} \times I\right) \subset T G_{r, s}, s$ sufficiently large. We may assume $L_{t}=\theta_{1}$ for $t$ near 1 , say $t \in[1-\eta, 1]$; then $L$ is differentiable and transverse to $G_{r, s}$ on $N_{\varepsilon}(M) \times[1-\eta, 1]$ since $\theta_{1}$ has this property on $N_{\varepsilon}(M)$. As in the proof that $\Theta$ is onto we may deform, $T f_{r} \circ L$ (relative to $\left.N_{\varepsilon} \times[1-\eta, 1]\right)$ in a neighborhood of $G_{r, s}$ to a map $G_{r}$ which is differentiable and transverse to $G_{r, s}$. Then $W=G_{r}^{-1}\left(G_{r, s}\right)$ is a submanifold of $E^{n+r} \times I$ with $\partial W=M$ and meeting $E^{n+r} \times 0$ perpendicularly. Again as above we may assume $G_{r} \mid W$ is the normal map, and $G_{r}$ on some normal tube of $W$ is translation of vectors to the origin. Covering this homotopy of $T f_{r} \circ L$ by a homotopy of $L$ to a map $\theta: S^{n+r} \times I \rightarrow T B_{r}, \theta_{t}=\theta_{1}$ for $t$ near 1 (and $\theta \mid S^{n+r} \times 0=\theta_{0}$.) Further, $\theta \mid W: W \rightarrow B_{r}$ covers the normal map $G_{r} \mid W$. Since $\theta_{1}$ covers the normal map of $M$, the $(B, f)$-structure defined on $W$ by $\theta$ induces the given $(B, f)$-structure on $M$. Hence $M$ is $(B, f)$-cobordant to zero. This shows that $\Theta$ is one-to-one, and completes the proof of the theorem.

The above proof was for bundle pairs $\left(B_{r}, f_{r}\right)$; but if $\left(C_{r}, h_{r}\right)$ is homotopically equivalent to $\left(B_{r}, f_{r}\right)$, the Thom spaces $T\left(h_{r}^{*}\left(\gamma^{r}\right)\right)$ are homotopically equivalent to $T\left(f_{r}^{*}\left(\gamma^{r}\right)\right)$, and the induced isomorphisms on homotopy commute with all the maps. Thus Theorem $\mathrm{C}$ follows immediately from the above theorem.

RemarK. In connection with the above argument, it should be noted that even if for example $T f_{r} \circ \theta_{1}$ is homotopic to the constant map, $\theta_{1}$ may not be 
so; because the homotopy of $T f_{r} \circ \theta_{1}$ may slide over the point at $\infty$, and this cannot always be covered by a homotopy of $\theta_{1}$.

3. Some special classifying spaces. Let $B U_{n}^{q}, n>q$, be the space obtained from $B U_{n}\left(U_{n}\right.$ the $n$-dimensional unitary group) by killing off the first $2 q-1$ homotopy groups (cf. Adams [1]). Then there is a natural map $g_{n}: B U_{n}^{q} \rightarrow B U_{n}$ which is an isomorphism on all higher homotopy groups. Since $B U_{n}$ is a countable CW-complex, we may choose $B U_{n}^{q} \in W_{0}$, [7] and hence by Proposition 2.1, $g_{n}$ to be a bundle projection.

Let $u \in H^{2 k}\left(B U_{n}^{q} ; Z\right), k<n$, and let $B U_{n}^{q}(\hat{u})$ be the fibre of a fibre map $\phi: B U_{n}^{q} \rightarrow K(Z, 2 k)$ with $\phi^{*} i_{2 k}=u$. Again we may assume $B U_{n}^{q}(\hat{u}) \in W_{0}$ and hence replace the inclusion by a bundle map $h_{n}: B U_{n}^{q}(\hat{u}) \rightarrow B U_{n}^{q}$.

Given a bundle with group $U_{n}$ over a polyhedron $X$, let $\psi: X \rightarrow B U_{n}$ be a classifying map. Then the bundle is trivial over the $2 q-1$ skeleton of $X$ if and only if $\psi$ may be lifted to a map $\hat{\psi}: X \rightarrow B U_{n}^{q}$. Given such a bundle, $\hat{\psi}$ may be further lifted to $B U_{n}^{q}(\hat{u})$ if and only if $\hat{\psi}^{*}(u)=0$.

We evidently may choose maps $j_{n}^{q}, \hat{j}_{n}^{q}$ to make a commutative diagram,

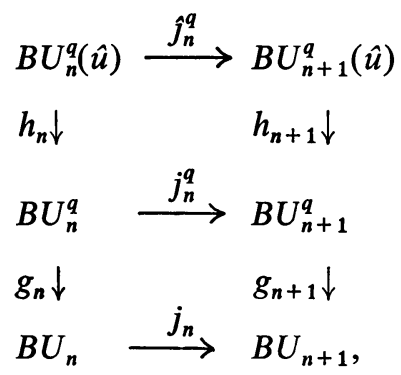

where $u \in H^{2 k}\left(B U_{n+1}^{q} ; Z\right)$ and by abuse of notation we use the same symbol for its image in $H^{2 k}\left(B U_{n}^{q} ; Z\right)$; such that $j_{n},{ }_{j n}^{q}, \hat{j}_{n}^{q}$ induces isomorphisms in homotopy up through dimension $2 n$. We thus have $H^{*}\left(B U_{\infty}^{q}\right) \simeq \operatorname{Lim} H^{*}\left(B U_{n}^{q}\right)$, similarly $H^{*}\left(B U_{\infty}^{q}(\hat{u})\right) \simeq \operatorname{Lim} H^{*}\left(B U_{n}^{q}(\hat{u})\right)$, arbitrary coefficients, where say $u \in H^{2 k}\left(B U_{\infty} ; Z\right)$ is any polynomial in the Chern classes (and we again use the same symbol for the image of $u$ under the various maps). Let $Q$ be the rational numbers.

Proposition 3.1. (a) $H^{*}\left(B U_{\infty}^{q} ; Q\right) \simeq Q\left[c_{q}, c_{q+1}, \cdots\right]$, where we again write $c_{i}$, for the image under the homomor phism $H^{*}\left(B U_{\infty} ; Z\right) \stackrel{g^{*}}{\rightarrow} H^{*}\left(B U_{\infty}^{q} ; Z\right) \rightarrow H^{*}\left(B U_{\infty}^{q} ; Q\right)$, of the ith Chern class.

(b) If $u$ is any polynomial in the $c_{i}$ 's, $h^{*}: H^{*}\left(B U_{\infty}^{q} ; Q\right) \rightarrow H^{*}\left(B U_{\infty}^{q}(\hat{u}) ; Q\right)$ is onto and has kernel the ideal generated by $u$.

Proof. $B U_{\infty}^{q}$ is the same homotopy type as $\Omega^{2} B U_{\infty}^{q+1}$ [1]. Since

$$
\pi_{i}\left(B U_{\infty}^{q}\right)= \begin{cases}Z & i=2 q+2 j, \quad j=0,1,2, \cdots \\ 0 & \text { otherwise }\end{cases}
$$


it follows from the theory of $H$-spaces [10] that the rational cohomology ring is a polynomial algebra over $Q$ with generators dual to the image in $H_{r}\left(B U_{\infty}^{q} ; Q\right)$ of a set of generators of $\pi_{i}\left(B U_{\infty}^{q}\right) \times Q, i=1,2, \cdots$. The result (a) follows from the known properties of $B U_{\infty}$ and the definition of the map $g: B U_{\infty}^{q} \rightarrow B U_{\infty}$.

To prove (b), we use the fact that $K(Z, 2 k-1)$ has the same rational cohomology as a sphere, and hence we can apply the Gysin sequence to the fibration

$$
K(Z, 2 k-1) \rightarrow B U_{\infty}^{q}(\hat{u}) \stackrel{h}{\rightarrow} B U_{\infty}^{q},
$$

and obtain the exact sequence (rational coefficients):

$$
\cdots \leftarrow H^{i}\left(B U_{\infty}^{q}(\hat{u})\right) \stackrel{h^{*}}{\leftarrow} H^{i}\left(B U_{\infty}^{q}\right) \stackrel{\rho}{\leftarrow} H^{i-2 k}\left(B U_{\infty}^{q}\right) \leftarrow \cdots,
$$

where $\rho$ is cup product with the class $u \in H^{2 k}\left(B U_{\infty}^{q}\right)$. The result (b) follows immediately.

A manifold $M^{n}$ is called weakly complex if the group of its normal bundle in some $E^{n+2 r}$ is reducible to $U_{r}$. In our terms a weakly complex manifold is a $(B U, f)$-manifold, where $f_{r}: B U_{r} \rightarrow B O_{2 r}$ is a bundle projection with fibre $O_{2 r} / U_{r}$. The $(B U, f)$ cobordism is called complex cobordism, and a $\left(B U_{r}, f_{r}\right)$-structure will also be called a $U_{r}$ structure. For convenience we will also refer to a $B U_{r}^{q}$ structure as a $U_{r}^{q}$ structure, etc. (i.e., $U_{r}^{q}$ may be considered the group whose universal base space is $B U_{r}^{q}$, as in $\S 1$ ).

As is well known, the map in homotopy

$$
\pi_{n+2 r}\left(T B U_{r}, \infty\right) \stackrel{\Sigma^{2}}{\longrightarrow} \pi_{n+2 r+2}\left(\Sigma^{2} T B U_{r}, \infty\right) \stackrel{T j_{r}}{\longrightarrow} \pi_{n+2 r+2}\left(T B U_{r+1}, \infty\right),
$$

is an isomorphism for $n<2 r$. Also Milnor [4] has shown that the Hurewicz map $\pi_{n+2 r}\left(T B U_{r}, \infty\right) \rightarrow H_{n+2 r}\left(T B U_{r}\right)$ is a monomorphism, $n<2 r$, and it follows that two weakly complex closed manifolds are complex cobordant if and only if they have the same Chern numbers.

Any normal map $\psi_{0}: M \rightarrow B O_{2 r}$ for the normal bundle of a weakly complex manifold $M$ may be lifted to $\psi: M \rightarrow B U_{r}$ by the $(B U, f)$-structure. The last statement in the above paragraph implies that the cobordism class of $M$ depends only on the homotopy class of $\psi$ in $B U_{r}$ (i.e., independent of a specific isotopy class of liftings within the homotopy class).

4. Poincaré duality for complex cobordism classes. Let $M^{n}$ be a closed weakly complex manifold whose normal bundle in a sufficently large Euclidean space is trivial over the $(2 q-1)$-skeleton. (This is always true for $q=1$.) Let $\psi: M \rightarrow B U$ (actually $B U_{2 r}$, for $r$ sufficiently large, but we omit the subscript hereafter) be a classifying map for the normal bundle and $\bar{\psi}: M \rightarrow B U^{q}$ any lifting. Let $u \in H^{2 k}(B U ; Z)$, and $u_{M}=\psi^{*}(u) \in H^{2 k}(M ; Z)$. Suppose $u_{M}$ is spherical; i.e., there exists a map $f: M \rightarrow S^{2 k}$, such that $f^{*} \gamma_{2 k}=u_{M}, \gamma_{2 k} \in H^{2 k}\left(S^{2 k} ; Z\right)$ a generator. 
Deforming $f$ to be differentiable and $t$-regular on the base point $p$ of $S^{2 k}$ we, have $f^{-1}(p)$ a submanifold $N^{n-2 k}$ imbedded in $M$. Then $N$ represents an homology class of $M$ dual to $u_{M}$, and a fortiori:

(1) Some nonzero multiple of the homology class of $M$ represented by $N$ is dual to $u_{M}$.

Also since the normal bundle of $N$ in $M$ is equivalent to the pull back of the normal bundle of $p$ in $S^{2 k}$, we have:

(2) The normal bundle of $N$ in $M$ is trivial.

Choosing a particular product structure for the normal bundle of $N$ in $M$, we see that the unitary structure of the normal bundle of $M$ in Euclidean space induces a $U \times I_{2 k}$-structure on the normal bundle of $N$ in Euclidean space, where $I_{2 k}$ is the subgroup of $O_{2 k}$ containing the unit element only. The classifying map for this structure is given by

$$
N \subset M \stackrel{\psi}{\rightarrow} B U=B\left(U \times I_{2 k}\right) .
$$

Proposition 4.1. Let $N \subset M$ satisfy (1) and (2) above. If every Chern number of $M$ having $u_{M}$ as a factor is zero, $N$ (with any $U \times I_{2 k}$ structure as defined above) is $U \times I_{2 k}$ cobordant to zero.

Proof. $T B\left(U_{r} \times I_{2 k}\right)=\Sigma^{2 k} T B U$ and $\pi_{n+2 r}\left(T B\left(U_{r} \times I_{2 k}\right)\right) \simeq \pi_{n-2 k+2 r}\left(T B U_{r}\right)$, $2 r>n$. It follows that the $U \times I_{2 k}$ cobordism classes are determined by their Chern numbers. If $v$ is any homogenous polynomial in the Chern classes, $v_{N}$ is the pull back of $v_{M}$, by the last remark above. Now let $v \in H^{n-2 k}(B U ; Z)$; then if $s N$ is dual to $u_{M}, s v_{N}[N]=u_{M} v_{M}[M]=0$ by hypothesis, and hence $v_{N}[N]=0$. Thus all Chern numbers of $N$ are zero, and $N$ is $U \times I_{2 k}$ cobordant to zero.

Given $N \subset M$ satisfying (1) and (2) above, we may choose a map $f: M \rightarrow S^{2 k}$ such that $f^{*} s \gamma_{2 k}=u_{M}$ and $f$ maps:

(a) The normal tube $N \times D^{2 k} \subset M$ into $S^{2 k}$, by first projecting onto $D^{2 k}$ and then by a relative homeomorphism $\left(D^{2 k}, S^{2 k-1}\right) \rightarrow\left(S^{2 k}, p^{\prime}\right), p^{\prime}$ the antipodal point to $p$, sending $o \in D^{2 k}$ into $p$.

(b) $M-\left(N \times \operatorname{Int} D^{2 k}\right)$ into $p^{\prime}$.

Proposition 4.2. Given $N \subset M$ satisfying (1) and (2) above, $\bar{\psi} \mid\left(M-N \times \operatorname{Int} D^{2 k}\right)$ can be lifted to $\hat{\psi}: M-\left(N \times \operatorname{Int} D^{2 k}\right) \rightarrow B U^{q}(\hat{u})$.

Proof. In fact the map $M \stackrel{\psi}{\rightarrow} B U^{q} \stackrel{g}{\rightarrow} K(Z, 2 k), g^{*} i_{2 k}=u$; is homotopic to the map $M \stackrel{f}{\rightarrow} S^{2 k} \stackrel{h}{\rightarrow} K(Z, 2 k), f$ as above, $h^{*} i_{2 k}=s \gamma_{2 k}$, since $\Psi^{*} g^{*} i_{2 k}=f^{*} h^{*} i_{2 k}=u_{M}$. By (b) above, $f$ is trivial on $M-N \times \operatorname{Int} D^{2 k}$ and $g \Psi \mid\left(M-N \times \operatorname{Int} D^{2 k}\right)$ is homotopically trivial. It follows that $\bar{\psi} \mid\left(M-N \times \operatorname{Int} D^{2 k}\right)$ can be factored up to homotopy through $B U^{q}(\hat{u})$. By the covering homotopy property, $\bar{\psi} \mid\left(M-N \times \operatorname{Int} D^{2 k}\right)$ may be lifted to $B U^{q}(\hat{u})$. 
Now assume $u_{M}$ satisfies the hypothesis of Proposition 4.1. We wish to construct a manifold $M_{1}$ cobordant to $M$, such that the classifying map $\psi_{1}$ of $M_{1}$ can be lifted on all of $M_{1}$ to $B U^{q}(\hat{u})$. The idea is to cut out the 'bad' part of $M$; namely, $N \times \operatorname{Int} D^{2 k}$; and attach $W \times S^{2 k-1}$ instead, where $\partial W=N$, and $W$ has the property that the lifting $\hat{\psi} \mid N \times D^{\circ 2 k}$ can be extended to $W \times S^{2 k-1}$. To begin with we need a construction due to Wall [15]:

Definition 4.3. Let $M^{n}$ be a closed $G$-manifold and $N^{n-r}$ a closed manifold with trivial normal bundle. Then any $G \times I_{r}$ cobordism of $N$ may be extended to a $G$-cobordism of $M$ as follows: Let $W$ be a $G \times I_{r}$ cobordism of $N$ with $N_{1}$. Attach $W \times D^{r}$ to $M^{n} \times I$ by identifying $N \times D^{r} \times(1)$ in $M^{n} \times I$ to $N \times D^{r}$ in $W \times D^{r}$, and smooth out the corners (see [15] for details). This gives a manifold $V^{n+1}=\left(M^{n} \times I\right) \cup\left(W \times D^{n}\right)$ with boundary the disjoint union of $M$ and a manifold $M_{1}=\left(M-N \times\right.$ Int $\left.D^{r}\right) \cup\left(W \times S^{r-1}\right) \cup\left(N_{1} \times D^{r}\right)$ (attached along the common boundaries and with corners smoothed). Since the structures agree where identified, $V^{n+1}$ may be given a $G$-structure so that the restriction to $M \subset V^{n+1}$ is the given one. Thus the $G$-structure on $M_{1}$, obtained by restriction, makes $M_{1}$ $G$-cobordant to $M$.

Finally, we remark that if $N_{1}$ is empty, $M_{1}=\left(M-N \times \operatorname{Int} D^{r}\right) \cup\left(W \times S^{r-1}\right)$. We call this the G-cobormism of $M$ defined by the $G \times I_{r}$ cobordism of $N$.

Proposition 4.4. Let $N^{n-2 k}$ be imbedded in a $U^{q}$-manifold $M^{n}, N$ and $M$ closed, such that

(1) Some nonzero multiple of $N$ represents an homology class in $M$ dual to $u_{M}$.

(2) $N$ has a trivial normal bundle in $M$.

(3) $N$ is connected.

(4) Giving $N=N \times p\left(p \in S^{2 k-1}=D^{\circ 2 k}\right)$ a $U^{q}(\hat{u}) \times I_{2 k}$ structure defined by $\hat{\psi} \mid N \times p\left(\hat{\psi}\right.$ as in 4.2), $N$ is $U^{q}(\hat{u}) \times I_{2 k}$ cobordant to zero.

Then $M$ is $U^{q}$-cobordant to a manifold $M_{1}$ with $u_{M_{1}}=0$.

Explicitly, if $W$ is a $U^{q}(\hat{u}) \times I_{2 k}$ manifold with boundary $N$, then $M_{1}=\left(M-N \times \operatorname{Int} D^{2 k}\right) \cup\left(W \times S^{2 k-1}\right)($ see 4.3$)$ is $U^{q}$-cobordant to $M$ and admits a $U^{q}(\hat{u})$-structure.

Proof. $W$ is a fortiori a $U^{q} \times I_{2 k}$ cobordism of $N$ to zero, and since all of $M$ has a $U^{q}$-structure, the construction of (4.3) yields a $U^{q}$-structure on $V^{n+1}$ and hence $M_{1}$. Let $\Psi_{1}: M_{1} \rightarrow B U^{q}$ define this structure; then we may assume that $\bar{\psi}_{1} \mid W \times S^{2 k-1}$ factors through $p_{1}: W \times S^{2 k-1} \rightarrow W$, since $\bar{\psi}_{1}$ may be extended to $V^{n+1}$ and hence over $W \times D^{2 k}$. Thus we have the commutative diagram:

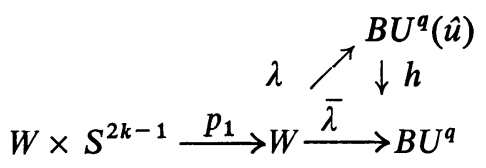

where $\bar{\lambda} p_{1}=\bar{\psi} \mid W \times S^{2 k-1}$ and $\lambda$ covers $\bar{\lambda}\left(\lambda\right.$ exists since $W$ is a $U^{o}(\hat{u}) \times I_{2 k}$ 
cobordism of $N$ to zero). Then $\lambda p_{1}$ restricted to $N \times S^{2 k-1}$ covers $\bar{\psi}\left|N \times S^{2 k-1}=\bar{\psi}_{1}\right| N \times S^{2 k-1}$, and we will see how this differs from $\hat{\psi} \mid N \times S^{2 k-1}$.

Now $h: B U(\hat{u}) \rightarrow B U^{q}$ may be assumed to be a principal bundle with group $K(Z, 2 k-1)$ (the bundle as defined in $\S 3$ is in any case of the same fibre homotopy type by [2]). Hence $\lambda p_{1}$ and $\hat{\psi}$ restricted to $N \times S^{2 k-1}$ differ by a map $\rho: N \times S^{2 k-1} \rightarrow K(Z, 2 k-1)$. The homotopy class of $\rho$ is determined by $\rho^{*} i_{2 k-1} \in H^{2 k-1}\left(N \otimes S^{2 k-1} ; Z\right)=H^{2 k-1}(N ; Z) \otimes H^{0}\left(S^{2 k-1} ; Z\right)+H^{2 k-1}\left(S^{2 k-1} ; Z\right)$ $\otimes H^{0}(N ; Z)$. But $\lambda p_{1}$ and $\hat{\psi}$ agree on $N \times p$; and hence if $N$ is connected, we may consider that $\rho^{*} i_{2 k-1} \in H^{2 k-1}\left(S^{2 k-1} ; Z\right)$. Then there exist a map $\theta: S^{2 k-1} \rightarrow K(Z, 2 k-1)$ with $\theta^{*} i_{2 k-1}=\rho^{*} i_{2 k-1}$. And

$$
W \times S^{2 k-1} \stackrel{\lambda \times \theta}{\longrightarrow} B U^{q}(\hat{u}) \times K(Z, 2 k-1) \stackrel{\mu}{\rightarrow} B U^{q}(\hat{u})
$$

( $\mu$ the action of $K(Z, 2 k-1)$ on $B U^{q}(\hat{u})$ ), belongs to the same homotopy class of liftings as $\hat{\psi} \mid N \times S^{2 k-1}$. Thus $M_{1}=\left(M-N \times \operatorname{Int} D^{2 k}\right) \cup\left(W \times S^{2 k-1}\right)$ may be given a $B U^{q}(\hat{u})$-structure.

We have yet to show under what circumstances conditions (3) and (4) of Proposition 4.4 are fulfilled. Condition (3) is taken care of as follows: First, any closed weakly complex manifold $M^{n}$ is complex cobordant to a connected manifold. Second, if $N^{n-s}, s>1$, is imbedded in a connected $M^{n}$ with a trivial normal bundle, $N$ is cobordant in $M$ to a connected manifold imbedded with trivial normal bundle. Although these two statements are easy to prove directly, we will not include the proofs here, as the statements are an immediate consequence of the zero-dimensional cases of Propositions 5.1 and 5.2 of $\S 5$.

We would now like to show that $N$ satisfying the hypothesis of (4.1) is $U^{q}(\hat{u}) \times I_{2 k}$ cobordant to zero. However, we can only show in general that some nonzero multiple $r$ of $N$ is $U^{q}(\hat{u}) \times I_{2 k}$ cobordant to zero (4.5) below. Since $N$ is imbedded in $M$ with trivial normal bundle, we may imbed $r$ disjoint copies of $N$ parallel to each other in a product neighborhood of $N$ in $M$, and hence the connected sum $r N$. Thus, starting with an $N \subset M$ satisfying the hypothesis of (4.1), we may replace it with an $N^{\prime} \subset M^{\prime}$ satisfying (1), (2), (3), (4) of (4.4), but with $r u$ in place of $u$ (note that $r N$ is a fortiori $U^{q}(r \hat{u}) \times I_{2 k}$ cobordant to zero).

Since (1) and (2) are satisfied if $u_{M}$ is spherical, Propositions 4.4 and 4.5 (below) imply.

THEOREM $\mathrm{A}^{\prime}$. Let $u_{M}$ be a homogenous polynomial in the Chern classes of a weakly complex closed manifold $M$. If $u_{M}$ is a spherical cohomology class and every Chern number of $M$ with $u_{M}$ as a factor is zero, then $M$ is complex cobordant to a manifold $M^{\prime}$, with $u_{M^{\prime}}$ of finite order. Further, if the normal bundle of $M$ is trivial over its $(2 q-1)$-skeleton, the same may be assumed for $M^{\prime}$ (in a sufficiently large Euclidean space). 
Corollary (TheORem A). Let $u_{M}$ be a homogeneous polynomial in the Chern classes of a weakly complex closed manifold $M$. If every Chern number of $M$ with $u_{M}$ as a factor is zero, $M$ is complex cobordant to a manifold $M^{\prime}$ with $u_{M^{\prime}}$ of finite order. Further, if the normal bundle of $M$ is trivial over its $(2 q-1)$ skeleton, the same may be assumed for $M^{\prime}$.

Proof. Assume first that $u_{M}^{2}$ is of finite order. Then some nonzero multiple $s$ of $u_{M}$ has its square zero. Hence, some nonzero multiple $t$ of $s u_{M}$ is spherical $\left({ }^{2}\right)$. By Theorem $\mathrm{A}^{\prime}, M$ is cobordant to $M^{\prime}$ with $t s u_{M^{\prime}}$ of finite order; i.e., $u_{M^{\prime}}$ is of finite order.

Now consider the general case. Since $M$ is finite-dimensional, some $2^{i}$ th power of $u_{M}$ is zero. If the hypothesis of the corollary holds for $u_{M}$ it holds for $u_{M}^{2 f}$. Thus by iterating the preceding argument $i-1$ times starting with $u_{M}^{2^{i-1}}$, we eventually get an $M^{\prime}$ cobordant to $M$ with $u_{M^{\prime}}$ of finite order.

Proposition 4.5. If a $\left(U^{q}(\hat{u}) \times I_{2 k}\right)$-manifold $N^{n-2 k}$ is $U \times I_{2 k}$ cobordant to zero, some nonzero multiple of $N$ is $U^{q}(\hat{u}) \times I_{2 k}$ cobordant to zero.

Proof. The $U \times I_{2 k}\left(U^{q}(\hat{u}) \times I_{2 k}\right)$-cobordism group of $n$-manifolds is isomorphic to $\pi_{n+2 r}\left(\Sigma^{2 k} T B U_{r}\right)\left(\pi_{n+2 r}\left(\Sigma^{2 k} T B U_{r}^{q}(\hat{u})\right)\right.$, if $r$ sufficiently large. The result will follow if we show that the map $\pi_{n+2 r}\left(\Sigma^{2 k} T B U_{r}^{q}(\hat{u})\right) \otimes Q \rightarrow \pi_{n+2 r}\left(\Sigma^{2 k} T B U_{r}\right) \otimes Q$ is a monomorphism.

Since we are only interested in the stable range, the Hurewicz map $\pi_{n+2 r}\left(\Sigma^{2 k} T B U_{r}\right) \otimes Q \rightarrow H_{n+2 r}\left(\Sigma^{2 k} T B U_{r}\right) \otimes Q=H_{n+2 r}\left(\Sigma^{2 k} T B U_{r} ; Q\right)$ is a monomorphism (similarly for $B U_{r}^{q}(\hat{u})$ ). Hence it is sufficient to show that $H_{n+2 r}\left(\Sigma^{2 k} T B U_{r}^{q}(\hat{u}) ; Q\right) \rightarrow H_{n+2 r}\left(\Sigma^{2 k} T B U_{r} ; Q\right)$ is a monomorphism; or $H_{n-2 k+2 r}\left(T B U_{r}^{q}(\hat{u}) ; Q\right) \rightarrow H_{n-2 k+2 r} \Sigma^{2 k}\left(T B U_{r} ; Q\right)$ is a monomorphism. Since our bundles are oriented, this is equivalent to $H_{n-2 k}\left(B U_{r}^{q}(\hat{u}) ; Q\right) \rightarrow H_{n-2 k}\left(B U_{r} ; Q\right)$ a monomorphism. Finally, this comes to showing that $H^{n-2 k}\left(B U_{r} ; Q\right)$ $\rightarrow H^{n-2 k}\left(B U_{r}^{q}(\hat{u}) ; Q\right)$ is onto. But this is proved in Proposition 3.1. Q.E.D.

\section{Spherical modifications of $(B, f)$-manifolds.}

Proposition 5.1. Let $M^{n}(n>1)$ be a $(B, f)$-manifold and $\gamma \in \pi_{k}\left(M^{n}\right), k<n / 2$. If $\psi_{*}(\gamma)=0, \psi: M^{n} \rightarrow B$ a classifying map for the normal bundle of $M$, then $M$ is $(B, f)$-cobordant to $M_{1}$, with $\pi_{K}\left(M_{1}\right) \simeq \pi_{K}(M) /(\gamma)((\gamma)$ a subgroup containing $\gamma$ ), and $\pi_{i}\left(M_{1}\right) \simeq \pi_{i}(M)$ for $i>k$.

Proof. We first note that a spherical modification (surgery) is the special case of the construction in (4.3) where $N=S^{k}$ and $W=D^{k+1}$. As pointed out in [9], $\gamma$ is represented by an imbedding $g: S^{k} \rightarrow M$, since $k<n / 2$. Since $\psi_{*}(\gamma)=0$, the normal bundle of $M$ restricted to $S^{k} \subset M$ is trivial, and since $k<n / 2$ the

(2) See for example: I. Berstein, Homotopy mod $C$ of spaces of category 2, Comment. Math. Helv. 35 (1961), 9-15 (Proposition 1.5). 
normal bundle of $S^{k}$ in $M$ must be trvial. It remains to show that if $M^{n}$ is a $G$-manifold $\left(G=\left(G_{r}\right), G_{r}\right.$ the group with universal base space $B_{r}$, see $\left.\S 1\right)$; we may choose a product structure on the normal bundle of $S^{k}$ in $M$, so that the $G \times I_{n-k}$ structure on $S^{k}$ may be extended over $D^{k+1}$.

We start with the fact that the $O_{r+n-k}$ structure on the normal bundle of $S^{k} \subset M \subset E^{n+r}$ may be lifted to a $G_{r} \times O_{n-k}$ structure. By what was observed above, this may be lifted to an $I_{r} \times O_{n-k}$ structure and thence to an $I_{r} \times I_{n-k}$ structure. Now an arbitrary choice of these product structures may not give a product structure on the normal bundle of $S^{k}$ in $E^{n+r}$ which is extendable to $D^{k+1}$, but it will differ at most by a map of $S^{k}$ into $O_{r+n-k}$ from such a structure. On the other hand, the choice of the lifting of the $O_{n-k}$ structure on the normal bundle of $S^{k}$ in $M$ to an $I_{n-k}$ structure may be varied by any map of $S^{k}$ intc $O_{n-k}$. But the inclusion map $i: O_{n-k} \rightarrow O_{r+n-k}$ induces an epimorphism $i_{*}: \pi_{k}\left(O_{n-k}\right) \rightarrow \pi_{k}\left(O_{r+n-k}\right)$, since $k<n-k$. It follows that we may choose an $I_{n-k}$ structure on the normal bundle of $S^{k}$ in $M$ so that the $I_{r} \times I_{n-k}$ structure, and hence a fortiori the $G_{r} \times I_{n-k}$ structure, on the normal bundle of $S^{k}$ in Euclidean space may be extended over $D^{k+1}$.

It now follows by (4.3) that $M^{n}$ is $B$-cobordant $((B, f)$-cobordant)) to the $G$ manifold $M_{1}=\left(M-S^{k} \times \operatorname{Int} D^{n-k}\right) \cup D^{k+1} \times S^{n-k-1}$. As pointed out in [9] $\pi_{k}\left(M_{1}\right)$ is a quotient of $\pi_{k}(M)$ by a subgroup containing $\gamma$, and $\pi_{i}\left(M_{1}\right) \simeq \pi_{i}(M)$ for $i<k$.

REMARK 1. If $k=0$, the conclusion of the proposition is interpreted to mean that $M_{1}$ has one less component than $M$. Since the spaces $\left(B_{r}, f_{r}\right)$ are assumed connected (see $\S 1$ ), we have the conclusion: Every $(B, f)$-manifold $M^{n}$ is $(B, f)$-cobordant to a connected manifold.

REMARK 2. The above argument that $M^{n}$ is $G$-cobordant to

$$
M_{1}=\left(M-S^{k} \times \operatorname{Int} D^{n-k}\right) \cup D^{k+1} \times S^{n-k-1}
$$

will hold if $n=4 s$ and $k=2 s$, since $i_{*}: \pi_{2 s}\left(O_{4 s}\right) \rightarrow \pi_{2 s}\left(O_{r+4 s}\right)$ is an epimorphism, provided $S^{k}$ has a trivial normal bundle in $M$.

Corollary. Let $M^{n}$ be a closed weakly complex manifold whose (stable) normal bundle is trivial over the $(2 r-1)$-skeleton and such that $u_{M}=0$, $u \in H^{2 k}(B U ; Z)$, then $M$ is complex cobordant to a manifold $M^{\prime}$ which is $\min (2 r-1,[(n+1) / 2], 2 k-2)$-connected, with $u_{M^{\prime}}=0$ and the normal bundle of $M^{\prime}$ trivial over the $(2 r-1)$-skeleton.

Proof. $M^{n}$ is a $B U^{r}(\hat{u})$ manifold. But $\pi_{i}\left(B U^{r}(\hat{u})\right)$ is zero if $i \leqq 2 r-1$ and $i \neq 2 k-1$. Hence the corollary follows from the proposition.

THEOREM B. Let $M^{n}$ be a closed weakly complex manifold with $c_{1}=c_{2}=\cdots=c_{k}=0,2 k<n / 2 ;$ then $M$ is complex cobordant to a $2 k$-connected manifold. 
Proof. We may assume $M$ is zero-connected. We prove inductively that $M$ is cobordant to a $2 i$-connected manifold with $c_{i+1}=\cdots=c_{k}=0$. But if $M$ is $2 i$-connected and $c_{i+1}=0$, the classifying map of the normal bundle of $M$, being trivial on the $2 i$-skeleton, may be assumed to send the boundaries of the $(2 i-1)$-cells into the base point. Since $\pi_{2 i+1}(B U)=0$, the classifying map will be trivial on the $(2 i+1)$-cells, and hence may be assumed to send the boundaries of the $(2 i+1)$-cells to the base point. But $c_{i+1}$ has a nonzero value on the generator of $\pi_{2 i+2}(B U)$, so the classifying map must be trivial on the $(2 i+2)$-skeleton. Thus the normal bundle restricted to $M^{(2 i+2)}$ is trivial. Thus $M$ is a $B U^{i+2}\left(\hat{c}_{i+2}, \cdots, \hat{c}_{k}\right)$-manifold, and is cobordant to a $(2 i+2)$-connected manifold with $c_{i+2}=\cdots=c_{k}=0$; just as in the corollary above, since $\pi_{j}\left(B U^{i+2}\left(\hat{c}_{i+2}, \cdots, \hat{c}_{k}\right)\right)=0, j<2 i+3$. Q.E.D.

Proposition 5.2. Let $M^{n}$ be a closed ( $\left.k-1\right)$-connected G-manifold, and let $N^{n-s}$ be a closed submanifold imbedded with trivial normal bundle. Then any spherical modification of $N$ to $N^{\prime}$ satisfying

(1) the modification is a $G \times I_{s}$ cobordism from $N$ to $N^{\prime}$,

(2) the modification is of type $(p+1, q+1), p+q+1=n-s$, where

can be made in $M$.

$$
p+1<\min (k+1, s, n / 2)
$$

Explicitly, if $W$ is the cobordism from $N$ to $N^{\prime}$ corresponding to the modification, then the imbedding of $N$ in $M$ may be extended to an imbedding of $W$ in $M$. Further, $W$ has a product structure on its normal bundle in $M$ which induces the given $G \times I_{s}$ structures on $N$ and $N^{\prime}$.

Proof. Let $f: S^{p} \times D^{q+1} \rightarrow N$ be an imbedding defining the modification. Since $M$ is $p$-connected, $S \stackrel{f_{0}}{\rightarrow} N \subset M$ is homotopic to a constant. Taking a normal frame on $N \subset M$, corresponding to the $I_{s}$ structure in (1), move $S^{p}$ a small distance in the direction of the first vector $\chi$ of this frame. Since $p+1<s$, we can get an imbedding of $D^{p+1}$ in $M$, with $N \cap D^{p+1}=S^{p}$ and such that $D^{p+1}$ comes into $N$ along this direction.

$N \subset M \subset E^{n+r}$ has a $G_{r} \times I_{s}$-structure on its normal bundle. Considering $E^{n+r} \subset E^{n+r+1}, N$ has an $I_{1} \times G_{r} \times I_{s}$ structure in $E^{n+r+1}$. In the two-dimensional subspace, of the normal space to each point of $N$, spanned by the vector $e$ perpendicular to $E^{n+r}$ and the first vector $\chi$ of the $I_{s}$ structure, rotate the frame $(e, \chi) 90^{\circ}$ so that $e$ comes into the position originally occupied by $\chi$. This does not change the $I_{1} \times G_{r} \times I_{s}$ structure on $N$ in $E^{n+r+1}$.

Now the $I_{q+1}$ structure on $S^{p}$ in $N$ together with the above, gives an $I_{1} \times G_{r} \times I_{s} \times I_{q+1}$ structure on $S^{p}$ in $E^{n+r+1}$. The fact that $f$ defines a $G \times I_{s}$ cobordism means that the $G_{r} \times I_{s} \times I_{q+1}$ structure on the normal bundle of $D^{p+1}$ in $E^{n+r+1}$ restricted to $S^{p}$, is extendable over $D^{p+1}$. On the other hand, the normal bundle of $D^{p+1}$ in $M$ has a unique (up to orientation) $I_{q+s}$ structure 
and this together with the $I_{1} \times G_{r}$ structure of $M$ in $E^{n+r+1}$ restricted to $D^{p+1}$, gives a $I_{1} \times G_{r} \times I_{q+s}$ structure on $D^{p+1}$ in $E^{n+r+1}$. Both of these structures come from the unique (up to orientation) $I_{q+r+s+1}$ structure on $D^{p+1}$ in $E^{n+r+1}$, by projection.

Now over $S^{p}$, these two structures are represented by frames which (if we choose the orientations properly) containing the same set of $r+1$ vector fields normal to $M$ over $S^{p}$. Consequently, the two $I_{q+s}$ structures on the normal bundle of $D^{p+1}$ in $M$ restricted to $S_{p}$ differ by a map $g: S_{p} \rightarrow O_{q+s}$ such that $S_{p} \stackrel{g}{\rightarrow} O_{q+s} \subset O_{q+s+r+1}$ is homotopic to the trivial map. Since $p<s-1$, we are in the stable range and $g$ is homotopic to the trivial map.

This means that we can extend the normal $I_{q+1}$-frame of $S^{p}$ in $N$ over $D^{p+1}$ and thus imbed $D^{p+1} \times D^{q+1}$ in $M$. Now thicken $N$ to $N \times I$ imbedded in $M$ using the vector field $-\chi$. Then we can imbed $N \times I \cup D^{p+1} \times D^{q+1}$ in $M$, and smooth the corners, so that $W$ is imbedded in $M$ and the $I_{s-1}$ structure on the nermal bundle of $N \times I$ in $M$ extends over $W$.

Since $W$ gives the $G \times I_{s}$ cobordism of $N$ to $N^{\prime}$ and induces the given $G \times I_{s}$ structure on $N$, we have $N^{\prime}$ imbedded in $M$ with an $I_{s}$ structure on the normal bundle. By using the reverse rotation, to that for $N$ above, on the corresponding pair of vectors in the normal bundle of $N^{\prime}$ in $E^{n+r+1}$, we see that the induced $G \times I_{s}$ structure is the same as that given by the cobordism.

Proposition 5.3. Let $M^{n}$ be a closed G-manifold and let $N^{n-s}$ be imbedded in $M$ with trivial normal bundle. Let $W$ taking $N$ to $N^{\prime}$ be a $G \times I_{s}$ cobordism corresponding to a spherical modification of $N$ of type $(p+1, q+1)$. Then $M^{\prime}=\left(M-N \times \operatorname{Int} D^{2 k}\right) \cup W \times S^{2 k-1} \cup N^{\prime} \times D^{2 k}($ see $\$ 4)$ is obtained from $M$ by a spherical modification of type $(p+1, q+s+1)$.

Proof. In fact $N^{\prime}=\left(N-S^{p} \times\right.$ Int $\left.D^{q+1}\right) \cup\left(D^{p+1} \times S^{q}\right), W=(N \times I)$ $\cup\left(D^{p+1} \times D^{q+1}\right)$. Hence $M^{\prime}-\left(S^{q} \times D^{2 k} \cup D^{q+1} \times S^{2 k-1}\right) \times$ Int $D^{p+1}=M-S^{p}$ $\times$ Int $\left(D^{q+1} \times D^{2 k}\right)$. Since in the construction of $\S 4$, the corners have been smoothed out, this is precisely a spherical modification of the stated type.

Note also that $M$ and $M^{\prime}$ make up the boundary of $V=(M \times I) \cup\left(W \times D^{2 k}\right)$, which is diffeomorphic to $(M \times I) \cup\left(D^{p+1} \times D^{q+2 k+1}\right)$.

Proposition 5.4. Let $M^{4 s}$ be a $(2 s-1)$-connected closed weakly complex manifold with $c_{s}^{2}=0$. If $s>1, M$ is complex cobordant to a $(2 s-1)$-connected manifold with $c_{s}=0$; and hence almost parallelizable.

Proof. Let $v \in H^{2 s}(M)$ be a generator such that $c_{s}$ is a multiple of $v$. Then $v^{2}=0$. The dual of $v$ is represented by $S^{2 s}$ imbedded in $M$ [9]. Since $c_{s}\left[S^{2 s}\right]=c_{s}, v_{s}=0$, the normal bundle of $M$ restricted to $S^{2 s}$ is trivial. It follows that the normal bundle of $S^{2 s}$ in $M$ may be given a product structure (see proof of Lemma 7 in [9]). Since $\pi_{2 s}\left(O_{2 s}\right) \rightarrow \pi_{2 s}\left(O_{r+2 s}\right)$ is onto, Remark 2 following 
Proposition 5.1, shows that we may choose a product structure on the normal bundle of $S^{2 s}$ in $M$ so that the corresponding spherical modification is a $U^{s}\left(\hat{c}_{s}\right) \times I_{2 s}$ cobordism; i.e., $D^{2 s+1}$ is a $U^{s}\left(\hat{c}_{s}\right) \times I_{2 s}$ cobordism of $S^{2 s}$ to zero. Thus the conditions of Proposition 4.4 are fulfilled and $M_{1}=\left(M-S^{2 s} \times \operatorname{Int} D^{2 s}\right) \cup D^{2 s+1} \times S^{2 s-1}$ is complex cobordant to $M$ and has $c_{s}=0$. As in the proof of Theorem 4 in [9], $M_{1}$ is $(2 s-1)$-connected. Q.E.D. Theorem $\mathrm{B}$ and the above proposition give:

THEOREM $\mathrm{B}^{\prime}$. Let $M^{4 s}, s>1$, be a closed weakly complex manifold with $c_{1}=c_{2}=\cdots=c_{s-1}=0$ and $c_{s}^{2}=0 ;$ then $M$ is complex cobordant to an almost parallelizable manifold.

A $\pi$-manifold is a manifold whose normal bundlein a sufficiently high dimensional Euclidean space is trivial; i.e., the group reduces to the identity element of the orthogonal group. We will call the correspoding cobordism theory, $I$ cobordism. Pontrjagin proved that the $I$ cobordism classes of $n$-dimensional closed manifolds forms a group (under disjoint union) isomorphic to $G_{n}=\pi_{m+n}\left(S^{m}\right), m>n+1$. This is of course a special case of the general cobordism theory of $\$ 2$. We now prove:

THEOREM C. If $n=4 k$, and $M^{n}$ is a closed $\pi$-manifold with product structure $\gamma$, then $\left(M^{n}, \gamma\right)$ is I-cobordant to $\left(M^{\prime}, \gamma^{\prime}\right)$, where $M^{\prime}$ is a differentiable n-manifold homeomorphic to a sphere and $\gamma^{\prime}$ is a product structure on $M^{\prime}$.

Proof. Since the universal base space of $I$ has all homotopy groups zero, Proposition 5.1 shows that $M$ is $I$-cobordant to a $(2 k-1)$-connected $\pi$-manifold. The same argument as in the proof of Theorem 4 in [9], together with Remark 2 following Proposition 5.1 (also see 5.4 above) shows that we may kill off the middle homotopy group to obtain a $2 k$-connected and hence $(4 k-1)$-connected $\pi$-manifold, at least for $k>1$. The theorem now follows by Smale's result [12] on the generalized Poincaré conjecture. (Since the stable 4-stem $\pi_{r+4}\left(S^{r}\right)$ is zero, the theorem is also true for $k=1$.)

\section{REFERENCES}

1. J. F. Adams, On Chern characters and the structure of the unitary group, Proc. Cambridge Philos. Soc. 57 (1961), 189-199.

2. E. Fadell, On fibre homotopy equivalence, Duke. Math. J. 26 (1959), 699-706.

3. M. Hirsch, Immersions of manifolds, Trans. Amer. Math. Soc. 93 (1959), 242-276.

4. J. Milnor, On the cobordism ring $\Omega^{*}$ and a complex analogue, Amer. J. Math. 82 (1960), 505-521.

5. - Construction of universal bundles. I, Ann. of Math. (2) 63 (1956), 272-284.

6. - Construction of universal bundles. II, Ann. of Math. (2) 63 (1956), 430-436.

7. - On spaces having the homotopy type of a CW-complex, Trans. Amer. Math. Soc. 90 (1959), 272-280. 
8. —, Differential topology, mimeographed, Princeton Univ., Princeton, N. J., 1958.

9. - Killing homotopy group of differentiable manifolds, Proc. Sympos. Pure Math.

Vol. 3, Differential Geometry, Amer. Math. Soc., Providence, R.I., 1961.

10. J. Milnor and J. Moore, On the structure of Hopf algebras, mimeographed, Princeton Univ., Princeton, N. J., 1959.

11. F. Peterson, Some remarks on Chern classes, Ann. of Math. (2) 69 (1959), 414-420.

12. S. Smale, Generalized Poincaré conjecture in dimensions greater than four, Ann. of Math.

(2) 74 (1961), 391-406.

13. N. Steenrod, The topology of fibre bundles, Princeton Univ. Press, Princeton, N.J., 1951.

14. R. Thom, Quelques propriétés globales des variétés différentiables, Comment. Math. Helv. 28 (1954), 17-86.

15. C. T. C. Wall, Cobordism of pairs, Comment Math. Helv. 35 (1961), 136-145.

16. M. Kervaire and J. Milnor, Groups of homotopy spheres. I, mimeographed notes: New York Univ., 1961.

UNIVERSTTY OF CHICAGO,

Chicago, Illinois 